\title{
ADAPTACIÓN TRANSCULTURAL Y VALIDACIÓN PSICOMÉTRICA DE INSTRUMENTO DE CALIDAD DE VIDA EN CUIDADOS PALIATIVOS
}

\author{
CROSS-CULTURAL ADAPTATION AND PSYCHOMETRIC VALIDATION \\ OF THE PALLIATIVE CARE QUALITY OF LIFE INSTRUMENT
}

\section{ADAPTAÇÃO TRANSCULTURAL E VALIDAÇÃO PSICOMÉTRICA DO INSTRUMENTO DE QUALIDADE DE VIDA EM CUIDADOS PALIATIVOS}

\author{
Antonia Vollrath Ramirez ${ }^{*}$ \\ Christine Bailey Catalan** \\ Antonia Arrate Vollrath ${ }^{* * *}$
}

\begin{abstract}
RESUMEN
La mayoría de los instrumentos de evaluación en salud han sido desarrollados en idioma anglosajón, lo que implica, para hispanohablantes, la realización de un proceso de adaptación transcultural (AT), que tiene aspectos y etapas esenciales como el rol del comité de expertos, la documentación detallada de los pasos y la prueba piloto de los instrumentos. Objetivo: Adaptar culturalmente y validar psicométricamente The Palliative Care Quality of Life Instrument ( $P Q L I)$ en pacientes chilenos con cáncer avanzado. Material y Método: Estudio descriptivo, transversal, en dos etapas. En etapa AT: traducción directa e inversa por traductores nativos independientes, análisis de expertos en cuidados paliativos y pretest a 20 pacientes de la unidad de cuidados paliativos de un hospital de la ciudad de Santiago de Chile, que cumplían como criterios: ser mayor de 18 años, alfabetizados y ser paciente con cáncer grado 1, 2 o 3 de acuerdo a la escala de estado funcional del Eastern Cooperative Oncology Group (ECOG). En la etapa de validación de las propiedades psicométricas se aplicó a un total de 155 pacientes, dando un Alfa de Cronbach de 0,806. Resultados: De la muestra, el 63,2\% de los pacientes tenía entre 50 a 69 años y el 56,8\% eran mujeres. Respecto a la selección de tratamiento, el 65,8\% se sintió capaz y dispuesto a participar, en tanto $P Q L I$ fue relacionada significativamente con el apoyo recibido de familiares y amigos. Acerca del apoyo del equipo de profesionales de salud, 92\% se siente apoyado, de lo cual $96 \%$ señala sentirlo respecto a las enfermeras. Conclusiones: Este instrumento adaptado permite conocer la opción de participar en la selección del tratamiento y tener una visión global de $P Q L I$ de las personas con cáncer avanzado en Chile.
\end{abstract}

Palabras clave: Calidad de vida; Neoplasia; Encuestas y Cuestionarios; Comparación Transcultural; Cuidados paliativos.

\footnotetext{
*Enfermera, PhD en Enfermería, Escuela de Enfermería, Facultad de Ciencias, Universidad Mayor, Santiago, Chile. ORCID: https://orcid.org/0000-0001-7652-7106 Email: maria.vollrath@umayor.cl Autora de correspondencia

**Socióloga, Magíster en Políticas Públicas. Dirección General de Vinculación con el Medio, Universidad de Playa Ancha, Valparaíso, Chile. ORCID: https://orcid.org/0000-0002-2814-8547 Email: christine.bailey@upla.cl

***Médica Cirujana, Facultad de Medicina, Universidad de Chile, Santiago, Chile. ORCID: https://orcid.org/0000-0003-36835037 Email: antonia.arrate@ug.uchile.cl
} 


\begin{abstract}
Most health assessment tools have been developed in the English language, which requires a cross-cultural adaptation process consisting of essential stages such as the role of the expert committee, detailed documentation of the steps and pilot testing. Objective: To culturally adapt and psychometrically validate the Palliative Care Quality of Life Instrument (PQLI) for Chilean patients with advanced cancer. Materials and Methods: Descriptive, cross-sectional, two-stage study. Cross-cultural adaptation stage: direct and back translation by independent native translators, analysis by experts in palliative care and pretest to 20 patients in the palliative care unit of a hospital in the city of Santiago de Chile, who met such criteria as being over 18 years old, literate and having cancer level 1, 2 or 3 according to the performance status scale of the Eastern Cooperative Oncology Group (ECOG). At the validation stage of psychometric properties, the instrument was applied to a total of 155 patients, resulting in a Cronbach's alpha of 0.806. Results: $63.2 \%$ of the patients were between 50 and 69 years old and 56.8\% were women. Regarding treatment selection, $65.8 \%$ felt able and willing to participate, while the PQLI was significantly related to the support received from family and friends. With respect to the support from the health professional team, 92\% felt supported; 96\% highlighted support from nurses. Conclusions: This adapted tool allows to know the option of participating in the selection of treatment and have an overview of the quality of life of people with advanced cancer in Chile.
\end{abstract}

Key words: Quality of Life; Neoplasms; Surveys and Questionnaires; Cross-cultural Comparison; Palliative Care.

\title{
RESUMO
}

A maioria dos instrumentos de avaliaçáo em saúde tem sido desenvolvidos na língua anglo-saxônica, o que requer um processo de adaptação transcultural (AT), que possui aspectos e etapas essenciais como atuação de um comitê de especialistas, documentaçáo detalhada das etapas e teste piloto. Objetivo: Adaptar culturalmente e validar psicometricamente The Palliative Care Quality of Life Instrument (PQLI) em pacientes chilenos com câncer avançado. Material e Método: Estudo descritivo, transversal, em duas etapas. Em etapa AT: tradução direta e inversa por tradutores nativos independentes, análise de especialistas em cuidados paliativos e pré-teste em 20 pacientes da unidade de cuidados paliativos do um hospital do Santiago do Chile, que cumpriram como critérios: ser maior de 18 anos, alfabetizados e ser um paciente com câncer de grau 1, 2 ou 3, de acordo com à escala de status de desempenho do Eastern Cooperative Oncology Group (ECOG). Na etapa de validação das propriedades psicométricas, foi aplicado a um total de 155 pacientes, resultando em um Alpha de Cronbach de 0,806. Resultados: Da amostra, o 63,2\% dos pacientes variaram entre 50 e 69 anos e o 56,8\% eram mulheres. Em relação à seleção de tratamento, 65,8\% sentiram-se aptos e dispostos a participar, enquanto a PQLI foi relacionada significativamente com o apoio que o paciente recebe de familiares e amigos. No que diz respeito ao apoio da equipe de profissionais de saúde, $92 \%$ sentem-se apoiados, dos quais $96 \%$ afirmam senti-lo em relação aos enfermeiros. Conclusóes: Este instrumento adaptado permite conhecer a opção de participar da seleção do tratamento e permite ter uma visão global da PQLI das pessoas com câncer avançado no Chile.

Palavras-chave: Qualidade de Vida; Neoplasias; Inquéritos e Questionários; Comparação Transcultural; Cuidados Paliativos.

Fecha de recepción: 09/10/2020

Fecha de aceptación: 03/06/2021

\section{INTRODUCCIÓN}

A nivel mundial, en el año 2018, el número de casos de pacientes con cáncer alcanza a 18,1 millones, mientras que la mortalidad relacionada con el cáncer alcanza 9,6 millones ${ }^{(1,2)}$. En Chile, durante el año 2018, el cáncer causó 28.443 muertes al año, correspondiendo a la segunda causa de muerte en el país, mientras otras cifras indicaban que el tipo de cáncer con más casos nuevos en mujeres era el 
cáncer de mamas $(20,8 \%)$ y en hombres, el cáncer de próstata $(23,9 \%)^{(3,4)}$.

Por otro lado, cabe mencionar que los efectos del cáncer en las personas producen cambios en la salud física y mental provocando estrés, trauma psicológico con sentimientos de depresión, temores al empeoramiento de la enfermedad, dudas sobre su supervivencia, entre otros efectos. Particularmente, en el caso de mujeres con cáncer de mama se suman problemas de autoestima e imagen corporal. Según lo anterior, se puede concluir que los efectos del cáncer impactan negativamente la calidad de vida de los pacientes ${ }^{(5,6)}$.

En este contexto, debemos tener en cuenta que la calidad de vida (CV) es un constructo compuesto por diversos componentes que varía según las culturas, épocas, grupos de edad y grupos sociales. Algunos autores definen la CV como una percepción subjetiva multidimensional determinada por condiciones que dependen de la percepción de las personas sobre la salud mental y física, satisfacciones personales, creencias, condiciones de su ambiente, apoyo social y familiar ${ }^{(7,8)}$. En relación con la medición de la $\mathrm{CV}$, en pacientes con cáncer, se ha vuelto cada vez más relevante dado el aumento de la supervivencia al cáncer $^{(9)}$ : en Australia, la supervivencia del cáncer de mama a 5 años es de 89,5\%, en los Estados Unidos alcanza un $90,2 \%$ y en Chile llega al $70 \%{ }^{(10)}$. Estos resultados representan un éxito, pero también conlleva nuevos desafíos para los profesionales de la salud, sobre cómo abordar las necesidades multidimensionales, aliviar el dolor y el sufrimiento, y mejorar la CV de pacientes y familias ${ }^{(11)}$. Además la medición de la CV se está considerando para evaluar avances $\mathrm{y}$ efectividad de tratamientos e intervenciones multidisciplinarias ${ }^{(12,13)}$.

Relacionado con lo anterior, se debe tener en cuenta que el $34 \%$ de los adultos que sufren de cáncer avanzado requieren cuidados paliativos $(\mathrm{CP})^{(14)}$. Dentro de este marco, cabe mencionar que en Chile está en vigencia el Programa de Alivio del Dolor por cáncer avanzado y Cuidados Paliativos, sus objetivos centrales son contribuir a mejorar la CV de personas con cáncer avanzado, unificar directrices y orientar al equipo de salud en los criterios de atención como evaluación de síntomas, el tratamiento tanto farmacológico como no farmacológico, la educación y otros aspectos como el bienestar emocional y la espiritualidad del paciente y su familia ${ }^{(15)}$. En este contexto, se han desarrollado diferentes herramientas para medir la $\mathrm{CV}^{(16)}$. En pacientes con cáncer, por ejemplo, están los cuestionarios European Organization for Research and Treatment of Cancer (EORTC), Core Quality of Life Questionnaire (EORTC QLQ-C30) y European Organization For Research And Treatment Of Cancer Head and Neck Cancer Quality of Life Questionnaire (EORTC QLQ-H\&N35) para evaluar el impacto de la quimioterapia ${ }^{(17)}$. A nivel nacional se utiliza Quality of Life Questionnaire Core 15 Palliative Care (EORTC QLQ C15 PAL) versión $1^{(18)}$, que es una versión abreviada del EORTC QLQ-C30, contiene subescalas para la evaluación de dominios como dolor, fatiga, náuseas y vómitos, entre otros, además de $\mathrm{CV}$ en forma global $^{(15)}$.

Es relevante mencionar que la mayoría de los instrumentos de evaluación en salud han sido desarrollados en idioma anglosajón, lo que implica un proceso de adaptación transcultural para ser utilizados en países de habla hispana. Dicho proceso tiene etapas esenciales, como la traducción inversa y la adaptación consensuada por un comité de expertos, la que es una etapa decisiva para establecer un rango de equivalencia aceptable para su uso, asegurando validez y confiabilidad del instrumento al ser aplicado en poblaciones con diversas culturas e idiomas. Por otro lado, es importante considerar que todo instrumento está en mayor o menor grado afectado por la cultura ${ }^{(19)}$, lo que en el área de la salud es especialmente relevante para conocer y comprender problemas sanitarios que trascienden las fronteras. Asimismo, la adaptación de un cuestionario de medición se ve determinado por las similitudes y diferencias en la estructura del lenguaje y la cultura entre la población para la cual se diseñó el instrumento y la población de destino ${ }^{(20)}$.

La adaptación transcultural (AT) tiene dos componentes esenciales: la traducción, que corresponde a la equivalencia semántica e implica que el significado de cada ítem traducido es el mismo en cada cultura; y la adaptación, es decir, la equivalencia de contenido, donde cada ítem del instrumento resulta relevante para describir el fenómeno estudiado en la población donde será aplicado, en este caso, el grupo de expertos y la prueba piloto. Así, la AT debe equilibrar la traducción literal de las palabras o frases y su adaptación, considerando el idioma, los giros idiomáticos y el contexto cultural de la población de destino, incluyendo también 
las diferencias en la percepción de los conceptos de salud y enfermedad de la población, los que también variarán puesto que están vinculados a su entorno cultural $^{(20,21)}$.

Sobre la base de la literatura revisada, se decide efectuar un estudio con el objetivo de realizar adaptación cultural y validación psicométrica del The Palliative Care Quality of Life Instrument (PQLI) en pacientes chilenos con cáncer avanzado. Entre las ventajas de este instrumento, por sobre otros utilizados en Chile, es que permite a los pacientes expresar su deseo de participar en la selección del tratamiento y brinda a los profesionales de la salud una visión biopsicosocial integral de la calidad de vida general desde la perspectiva del paciente.

\section{MATERIALES Y MÉTODO}

La investigación es de tipo descriptiva transversal que se llevó a cabo a través de una metodología caracterizada en 2 etapas ${ }^{(22,23)}$ : Etapa 1: En la AT del instrumento se realizó equivalencia semántica con la traducción directa e inversa de la versión original en inglés del PQLI. Dos hablantes nativos angloparlantes realizaron traducciones independientes al idioma español usado en Chile; en tanto, la traducción del instrumento fue realizada por un profesional chileno de lengua materna español y bilingüe con lengua materna inglés. Reuniendo ambos resultados se realizó una primera versión del instrumento en idioma español. Posteriormente, se realizó la equivalencia de contenido con participación de un grupo de expertos constituido por 8 profesionales de distintas instituciones gubernamentales, instituciones de salud y académicas vinculadas al ámbito oncológico y de CP, los cuales dieron su opinión especializa sobre el instrumento PQLI. Mediante este proceso se comprobó si los diferentes ítems y dimensiones de la primera versión del instrumento permitían medir los constructos o dominios originales, además de evaluar si el lenguaje empleado se adaptaba a la población de pacientes oncológicos en la que se pretendía aplicar, es decir, consistencia entre la comprensión de las preguntas y la pertinencia de las opciones de respuesta. Como resultado de ello, se anexaron los aportes sistematizados de los expertos, los que permitieron afinar una segunda versión consensuada del instrumento. Esta versión del instrumento se transformó en la prueba piloto (pretest), cuyo objetivo fue evaluar la equivalencia de contenido con la versión original. La prueba piloto se aplicó a 20 pacientes ${ }^{(23)}$ con cáncer avanzado de la unidad de CP y las principales conclusiones fueron que debido al estado letárgico de la mayoría de los pacientes, este cuestionario -cuyo tiempo promedio de aplicación fue $10 \mathrm{~min} \pm 5$ - debía ser aplicado por un entrevistador, en un lugar silencioso y tranquilo, e idealmente de manera privada sin acompañantes. En esta etapa, además, se modificaron 10 ítems del cuestionario, conservando las propiedades psicométricas, lo que dio como resultado un cuestionario equivalente al original $^{(22,24)}$. Una vez incorporados esos detalles, surgió la última versión adaptada. Etapa 2: Esta etapa de validación de propiedades psicométricas sirvió para confirmar la versión final adaptada del instrumento. Para verificar la validez de constructo se realizó un análisis factorial e Índice de Correlación Intraclase (ICC), mientras que para la confiabilidad se estimó el guarismo estadístico Alfa de Cronbach $(>0,7)^{(25)}$.

Participantes. El tamaño muestral se basó en la recomendación de Norman y Streiner ${ }^{(26)}$. La muestra se definió de acuerdo a cinco participantes respecto al número de ítems del cuestionario validado, siendo estos últimos 28 . Por tanto, el mínimo recomendado para establecer la validez factorial del cuestionario fue 140 participantes, a lo que se añadió el $10 \%$ para reponer la posible atrición o pérdida de participantes ${ }^{(26)}$. Se seleccionó a los participantes según los criterios de inclusión establecidos: ser mayor de 18 años, saber leer y escribir, ser un paciente con cáncer avanzado (grado 1, 2 o 3) de acuerdo a la Escala de Estado Funcional $(\mathrm{ECOG})^{(27)}$ y que fueran atendidos en la Unidad de Cuidados Paliativos de un hospital público de Santiago, Chile.

La ECOG permitió la siguiente categorización: Grado 1) Restringido en una actividad físicamente extenuado pero ambulatorio, capaz de realizar trabajos de naturaleza ligera o sedentaria, como labores ligeras en casa y trabajo de oficina; Grado 2) Ambulatorio y capaz de realizar todos los cuidados personales pero no puede realizar ninguna actividad laboral, se encuentra despierto y aproximadamente más del 50\% de las horas de vigilia; Grado 3) Capaz de autocuidado limitado, confinado a la cama o silla más del $50 \%$ de las horas de vigilia. 
Recolección de datos: El instrumento se aplicó, entre los meses de enero y febrero del año 2017, a un total de 155 pacientes y no estaban incluidos los 20 pacientes participantes en el pretest. Debido al estado físico letárgico de la mayoría de los pacientes, la entrevista fue realizada por estudiante de medicina capacitado para ese fin. Cada entrevista duró entre 10 y 15 min y se llevó a cabo en una sala privada y tranquila. Se leyó el formulario de consentimiento informado y todos los participantes firmaron.

La versión final de PQLI consta de 28 ítems y comprende 7 escalas de los dominios: 1) Estado de salud, 2) Cuidado personal, 3) Actividad, 4) Comunicación, 5) Apoyo, 6) Afección psicológica y 7) Opción de tratamiento. Éstas se responden con 3 respuestas posibles: $1=\mathrm{No}, 2=\mathrm{A}$ veces y $3=\mathrm{Si}$. Además, en Opción de tratamiento deben elegir "el elemento que es más importante del tratamiento para usted", calificándolo como 1 y "el elemento que es menos importante para usted”, calificándolo como 5 .

En cuanto a la evaluación de la Calidad de vida general, se utilizó una escala numérica de 0 a 10 , donde $0=$ malo y $10=$ excelente.

Procesamiento y análisis de datos. Para el plan de análisis psicométrico, el procesamiento de los datos fue realizado con el programa SPSS v.22. Se aplicó un factor multiplicador a los resultados para establecer una media de 0 a 100 para cada escala que compone el instrumento. De esta manera, una puntuación media más alta en la escala representa un estado de salud y una de sintomatología más baja. Además, se evaluaron las relaciones entre las variables sociodemográficas y las preguntas relacionadas con la salud de los participantes.

Para establecer la confiabilidad del instrumento (consistencia interna), se utilizó el Alfa de Cronbach $^{(28)}$. Debido a la naturaleza de la pregunta relacionada con la elección del tratamiento, el procedimiento para establecer la coherencia en esta sección se estableció mediante el coeficiente de concordancia y para este propósito se utilizó la prueba W de Kendall(29).

Para validar el instrumento se realizó un análisis factorial confirmatorio, dada su validación previa en otros contextos culturales ${ }^{(23)}$. Para esto se eligieron $\mathrm{y}$ analizaron 7 factores principales utilizando las pruebas de Kaiser-Meyer-Olkin (KMO) y Bartlett ${ }^{30,}$
31). Finalmente, los resultados se analizaron por tema, mapeando los datos para mejorar el rigor, la confiabilidad del análisis y los resultados del estudio. Este estudio fue aprobado por el Comité de Ética Científica de la Facultad de Enfermería de la Universidad Andrés Bello (código UNAB (L2/CECENF/10) en abril de 2016. Se informó a los autores del instrumento $P Q L I$, por vía correo electrónico, que se realizaría adaptación y validación en población chilena con cáncer avanzado.

\section{RESULTADOS}

Respecto a los indicadores demográficos, el 56,8\% de los entrevistados correspondía a mujeres; el $63,2 \%$ tenía entre 50 y 69 años; el 47,7\% de los pacientes tenía más de 12 años de escolaridad; el diagnóstico de cáncer más frecuente fue órganos digestivos (27,7\%); más del $60 \%$ de los entrevistados había sido diagnosticado hace más de un año antes del estudio y el 93,2\% de ellos, contaba con un miembro de la familia como su cuidador permanente (Tabla 1).

Validez: En la matriz de correlación, entre las subescalas PQLI, se observaron correlaciones moderadas de Pearson y las más fuertes se encontraron entre el Afecto psicológico y Estado de salud $(\mathrm{p}<0,01)$ y el Afecto psicológico y Calidad de vida $(\mathrm{p}<0,01)$. El dominio Actividad se relacionó con Estado de salud, Autocuidado, Calidad de vida y Afecto psicológico $(\mathrm{p}<0,01)$ (Tabla 2).

Confiabilidad: Se estableció utilizando el Alfa de Cronbach $^{(28)}$ (Tabla 3). En el análisis de la consistencia interna se obtiene un Alfa de Cronbach global de 0,805, mientras que el Alfa de Cronbach para cada subescala fue mayor que el valor crítico de 0,70 , demostrando confiabilidad y homogeneidad del cuestionario. Al detallar el Alfa para cada subescala, la consistencia u homogeneidad interna tendía a variar entre los ítems cuyos valores variaban entre 0 y 1 , considerando como aceptables aquellos valores iguales o mayores a $0,70^{(28,30)}$, tales como el Estado de salud $(0,828)$, Elección de tratamiento $(0,738)$, Comunicación $(0,740)$ y Afecto psicológico $(0,728)$. Estos resultados están de acuerdo con el $P Q L I$ original que mostró un alfa general de Cronbach de 0,787 ${ }^{(24)}$. 
Tabla 1. Variables sociodemográficas y antecedentes de salud, $2017(\mathrm{n}=155)$.

\begin{tabular}{|c|c|c|c|}
\hline Variables & & Fr & $\%$ \\
\hline \multirow[t]{2}{*}{ Sexo } & Hombre & 67 & 43,2 \\
\hline & Mujer & 88 & 56,8 \\
\hline \multirow[t]{3}{*}{ Edad } & 29-49 años & 28 & 18,1 \\
\hline & 50-69 años & 98 & 63,2 \\
\hline & 70-90 años & 29 & 18,7 \\
\hline \multirow[t]{3}{*}{ Nivel Educativo } & Estudios básicos & 43 & 27,7 \\
\hline & Estudios medios & 38 & 24,5 \\
\hline & Estudios superiores & 74 & 47,7 \\
\hline \multirow[t]{2}{*}{ Estatus de Convivencia } & Con pareja & 90 & 58,1 \\
\hline & Sin pareja & 65 & 41,9 \\
\hline \multirow[t]{2}{*}{ Composición del hogar } & Vive acompañado & 133 & 85,8 \\
\hline & Vive solo & 22 & 14,2 \\
\hline \multirow[t]{5}{*}{ Diagnóstico de Cáncer } & Órganos digestivos & 43 & 27,7 \\
\hline & Órganos genitales & 27 & 17,4 \\
\hline & Mama & 23 & 14,8 \\
\hline & Mieloma múltiple & 18 & 11,6 \\
\hline & Otros & 44 & 28,39 \\
\hline \multirow[t]{3}{*}{ Tiempo de diagnóstico } & $\mathrm{Al}$ menos 12 meses & 55 & 35,5 \\
\hline & 13 meses a 5 años & 67 & 43,2 \\
\hline & Más de 5 años & 33 & 21,3 \\
\hline \multirow[t]{2}{*}{ Se atiende en centro de Atención primaria } & Sí & 106 & 68,4 \\
\hline & No & 49 & 31,6 \\
\hline \multirow[t]{2}{*}{ Tiene cuidador } & Sí & 118 & 76,1 \\
\hline & No & 37 & 23,9 \\
\hline \multirow[t]{3}{*}{ Cuidador } & Familia & 147 & 94,8 \\
\hline & Amigos & 4 & 2,58 \\
\hline & Cuidador particular & 4 & 2,58 \\
\hline \multirow[t]{2}{*}{ Tipo de cuidado } & Permanente & 139 & 89,67 \\
\hline & Ocasional o transitorio & 16 & 10,32 \\
\hline
\end{tabular}

Tabla 2. Matriz de correlaciones entre dimensiones The Palliative Care Quality of Life Instrument (PQLI).

\begin{tabular}{lcccccccc}
\hline PQLI subescalas & AC & CP & ES & OT & A & CO & AP & QoL \\
\hline Actividad (AC) & & $0,436^{* *}$ & $0,461^{* *}$ & 0,073 & 0,144 & 0,068 & $0,458^{* *}$ & $0,451^{* *}$ \\
Cuidado personal (CP) & $0,436^{* *}$ & & $0,294^{* *}$ & $0,186^{*}$ & 0,114 & 0,078 & $0,348^{* *}$ & $0,373^{* *}$ \\
Estado de Salud (ES) & $0,461^{* *}$ & $0,294^{* *}$ & & $-0,003$ & $0,300^{* *}$ & 0,049 & $0,574^{* *}$ & $0,401^{* *}$ \\
Opción de Tratamiento (OT) & 0,073 & $0,186^{*}$ & $-0,003$ & & $-0,226$ & 0,083 & $-0,053$ & 0,074 \\
Apoyo (A) & 0,144 & 0,114 & $0,300^{* *}$ & $-0,226$ & & 0,019 & $0,328^{* *}$ & $0,324^{* *}$ \\
Comunicación (CO) & 0,068 & 0,078 & 0,049 & 0,083 & 0,019 & & $-0,059$ & 0,021 \\
Afección Psicológica (AP) & $0,458^{* *}$ & $0,348^{* *}$ & $0,574^{* *}$ & $-0,053$ & $0,328^{* *}$ & $-0,059$ & & $0,528^{* *}$ \\
Calidad de Vida (CV) & $0,451^{* *}$ & $0,373^{* *}$ & $0,401^{* *}$ & 0,074 & $0,324^{* *}$ & 0,021 & $0,528^{* *}$ & \\
\hline
\end{tabular}

* Correlación positiva, estadísticamente significativa $(\mathrm{p}<0,05)$.

** Correlación positiva, estadísticamente significativa $(\mathrm{p}<0,01)$. 
Tabla 3. Puntajes medios de las subescalas y coeficiente Alfa de Cronbach.

\begin{tabular}{lccc}
\hline Subescala & Media 1 & Desv. típ. & Alfa de Cronbach \\
\hline Actividad & 72,2954 & 18,39215 & 0,722 \\
Cuidado Personal & 73,6024 & 18,43966 & 0,736 \\
Estado de Salud & 83,6332 & 18,96298 & 0,836 \\
Opción de Tratamiento & 80,7443 & 18,92339 & 0,807 \\
Apoyo & 97,7028 & 19,444000 & 0,98 \\
Comunicación & 73,9759 & 18,46878 & 0,74 \\
Afección Psicológica & 81,9883 & 18,90888 & 0,819 \\
\hline Alfa de C Global & & & 0,805 \\
\hline
\end{tabular}

Análisis factorial: Después de evaluar la relevancia del análisis factorial confirmatorio, la correlación entre las variables fue moderada. La prueba de esfericidad de Bartlett $^{(30,31)}$ fue 1223.815, con una significación de $\mathrm{p}<0,001$. Para evaluar el ajuste de los puntajes para factores específicos, se realizó la rotación varimax ${ }^{(32)}$, los valores inferiores a 0,3 fueron suprimidos. La adecuación de la medición de la prueba $K M O$ fue igual a 0,781 , lo que demuestra la viabilidad de realizar el análisis factorial. La extracción del factor del eje principal se utilizó para analizar la estructura subyacente del cuestionario, que arrojó 7 factores independientes que representan el 59,42\% de la varianza (Tabla 4).

Se identificaron los mismos 7 factores sin ninguna diferencia material, como lo confirman los resultados de la rotación no ortogonal: Factor 1: Actividad (seguir trabajando, tareas domésticas, diversión, pasatiempos); Factor 2: Cuidado personal (conducción o transporte, autónomo); Factor 3: Estado de salud (dolor, náuseas / vómitos, falta de apetito, debilidad y/o cansancio, disnea, trastornos de las heces, trastornos del sueño); Factor 4: Opción de tratamiento (me gusta elegir, poder elegir); Factor 5: Apoyo (familiares y/o amigos, equipo de atención médica, personal de enfermería); Factor 6: Comunicación (relaciones sociales, problemas económicos y/o profesionales, problemas familiares); Factor 7: Afección psicológica (calma, optimismo, azul, control de la situación, miedo a la muerte). La escala general de calidad de vida no estuvo representada en ningún factor. Las puntuaciones más altas obtenidas fueron en los dominios Actividad, Autocuidado, Apoyo, Comunicación y Afección psicológica, lo que indica un mejor nivel de funcionamiento y calidad de vida. Sin embargo, una puntuación más alta en el dominio del Estado de salud indicó una mayor sintomatología y una peor calidad de vida. Debido al tipo de escala utilizada para la elección del dominio del tratamiento, la confiabilidad se calculó realizando la prueba de coeficiente de concordancia de Kendall y se encontró un valor de 0,344 ( $\mathrm{p}<$ $0,01)$.

Descripción de resultados por dominios: Se indican a continuación los resultados en puntuaciones de porcentajes para cada dominio: En Estado de salud, el 65,2\% de los entrevistados experimenta náuseas y vómitos y, a pesar de estar con tratamiento farmacológico, el 20\% de ellos experimenta dolor crónico y el $50 \%$ informa falta de apetito, problemas para dormir y se siente cansado; en Comunicación, cerca del $73 \%$ de los entrevistados no discute sus problemas familiares ni económicos con su médico; en Opción del tratamiento, se ha verificado que el $65,8 \%$ se siente capaz y desea participar en la elección del tratamiento; en tanto en el factor Apoyo, más del 90\% de los pacientes de la muestra se siente satisfecho con el soporte que ha recibido de parte de familiares, amigos y por el equipo de profesionales de salud, mientras el $96 \%$ se siente satisfecho con el apoyo brindado por las enfermeras de la unidad; en Afección psicológica, sobre el 60\% de los participantes se siente optimista pero también teme a la muerte; respecto a Actividad, se observó que el 66,5\% dejó de trabajar; Cabe destacar que uno de los hallazgos importantes fue el dominio Cuidado personal, donde el 90\% de ellos manifestó sentirse autónomo; respecto a la Calidad de vida, el 44\% obtuvo una puntuación entre 7 y 8, que corresponde a buena (Tabla 5). 


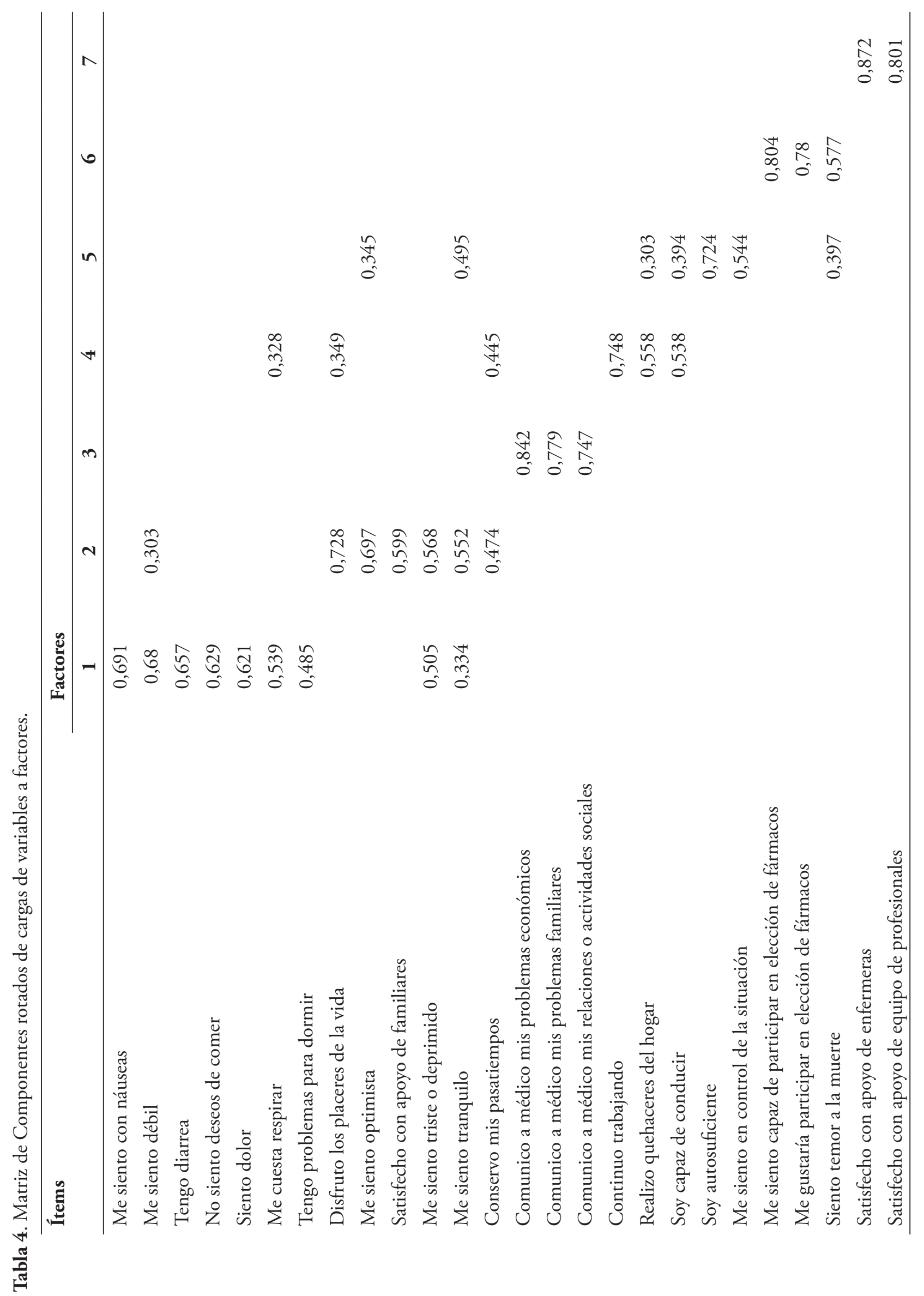


Tabla 5. Descripción por dominios (\%).

\begin{tabular}{|c|c|c|c|c|}
\hline Dominios & & No & $\mathrm{A} / \mathrm{V}$ & Si \\
\hline \multirow[t]{4}{*}{ Actividad (AC) } & $\begin{array}{l}\text { Continúo trabajando ((Considere trabajo remunerado, } \\
\text { voluntario) }\end{array}$ & 66,5 & 3,9 & 29,7 \\
\hline & Realizo quehaceres o labores del hogar & 18,7 & 15,5 & 65,8 \\
\hline & $\begin{array}{l}\text { Disfruto los placeres de la vida, lo que tú consideras el } \\
\text { placer de la vida. }\end{array}$ & 16,8 & 23,2 & 60,0 \\
\hline & $\begin{array}{l}\text { Conservo mis pasatiempos o actividades de tiempo } \\
\text { libre }\end{array}$ & 29,7 & 20,6 & 49,7 \\
\hline \multirow[t]{2}{*}{ Cuidado Personal (CP) } & $\begin{array}{l}\text { Soy capaz de conducir o utilizar medios de transporte } \\
\text { públicos }\end{array}$ & 24,5 & 3,2 & 72,3 \\
\hline & $\begin{array}{l}\text { Soy autosuficiente. Por ejemplo, puedo vestirme y } \\
\text { lavarme sin ayuda. }\end{array}$ & 6,5 & 3,2 & 90,3 \\
\hline \multirow[t]{7}{*}{ Estado de Salud (ES) } & Siento dolor & 49,7 & 30,3 & 20,0 \\
\hline & Me siento con náuseas, asco y/o vómitos & 21,9 & 12,9 & 65,2 \\
\hline & No siento deseos de comer & 25,8 & 24,5 & 49,7 \\
\hline & Me siento débil y cansado & 49,0 & 35,5 & 15,5 \\
\hline & Me cuesta respirar, siento que me ahogo & 15,5 & 19,4 & 65,2 \\
\hline & Tengo diarrea o estitiquez & 36,8 & 18,7 & 44,5 \\
\hline & $\begin{array}{l}\text { Tengo problemas para dormir (tomando o no pastillas } \\
\text { para dormir) }\end{array}$ & 41,3 & 10,3 & 48,4 \\
\hline \multirow[t]{3}{*}{ Apoyo (A) } & Familiares y/o amigos & 7,1 & 2,6 & 90,3 \\
\hline & Equipo de profesionales de salud que me atienden & 0,6 & 7,1 & 92,3 \\
\hline & Equipo de enfermeras que me atienden & 0,6 & 3,2 & 96,1 \\
\hline \multirow{3}{*}{$\begin{array}{l}\text { Comunicación (CO) "Converso } \\
\text { con el doctor de" }\end{array}$} & Mis relaciones o actividades sociales & 50,3 & 7,7 & 41,9 \\
\hline & Mis problemas económicos y profesionales & 76,1 & 7,1 & 16,8 \\
\hline & Mis problemas familiares & 72,9 & 9,7 & 17,4 \\
\hline \multirow[t]{5}{*}{ Afección Psicológica (AP) } & Me siento tranquilo & 13,5 & 16,8 & 69,7 \\
\hline & Me siento optimista & 18,1 & 13,5 & 68,4 \\
\hline & Me siento triste o deprimido & 21,3 & 40,0 & 38,7 \\
\hline & $\begin{array}{l}\text { Me siento en control de la situación de salud que } \\
\text { atravieso }\end{array}$ & 18,1 & 13,5 & 68,4 \\
\hline & Siento temor a la muerte & 30,3 & 3,2 & 66,5 \\
\hline \multirow[t]{2}{*}{ Opción de Tratamiento (OT) } & Me gustaría participar en la elección de mis fármacos & 43,2 & 3,9 & 52,9 \\
\hline & $\begin{array}{l}\text { Me siento capaz de participar en la elección de los tipos } \\
\text { de fármacos que necesito }\end{array}$ & 31,6 & 2,6 & 65,8 \\
\hline
\end{tabular}

\section{DISCUSIÓN}

En la literatura examinada, algunos autores ${ }^{(22,23)}$ señalan que actualmente en el mundo globalizado es cada vez más relevante contar con instrumentos de medición transversales adaptados y validados a las diversas culturas, que permitan comparar e intercambiar experiencias, buscar propuestas y recomendaciones para la construcción de protocolos clínicos, guiados por la evidencia científica y dando respuesta a problemas del ámbito de la salud. En este marco, la obtención de mediciones confiables y válidas permite a los profesionales de salud mejorar la calidad de atención, aumentar la satisfacción 
de los pacientes y obtener una mejor adherencia al tratamiento ${ }^{(22,33)}$. Por otra parte, es importante considerar que el proceso de traducción y adaptación de cuestionarios en países con idiomas y culturas diferentes, está en gran medida determinado por la cultura, por lo que este proceso debe ir secundado por un proceso de validación riguroso. Cabe destacar que en este proceso es crucial el consenso y recomendaciones de equivalencia del comité de expertos $^{(19,22)}$. En este sentido, en la realización de la AT y validación de un instrumento se requiere de un proceso de investigación riguroso, con una planificación y diseńo metodológico con respaldo científico para obtener hallazgos con fiabilidad y validez $^{(34)}$. En cuanto a los instrumentos adaptados y validados que valoran la calidad de vida relacionada con la salud, éstos permiten medir percepciones de las personas sobre el impacto de intervenciones o tratamientos que tienen sobre la calidad de vida y salud. También posibilitan pesquisar en una etapa temprana cualquier descompensación en aquellos grupos de riesgo, proporcionando una guía clínica a los profesionales de salud en intervenciones y/o tratamiento tanto en el proceso como en los resultados esperados ${ }^{(35,36)}$.

En suma, es posible establecer que el instrumento adaptado es coherente con el original de Mystakidou et al. ${ }^{(24)}$, y resultó ser de fácil ejecución y rapidez, requirió de 10 a 15 min, y fue bien aceptado por los pacientes. A este punto, es importante señalar que el cuestionario original es autoaplicado ${ }^{(24)}$ y que la versión del presente estudio se aplicó a través de entrevista individual, puesto que se consideró que las condiciones de salud de aletargamiento de los participantes lo hacían aconsejable. Por otra parte, cabe destacar que existe acuerdo en que los pacientes estaban agradecidos por el interés en medir su calidad de vida. Asimismo, hubo concordancia en que fue una oportunidad para que los pacientes participaran activamente, que tuvo una excelente aceptación la opción de participar en la elección del tratamiento, y la relevancia de la comunicación y el apoyo del equipo de profesionales con los pacientes y sus familias $^{(24)}$.

Particularmente, destacamos hallazgos del presente estudio en el dominio Apoyo, donde los resultados mostraron que la calidad de vida estaba muy relacionada con el apoyo recibido de la familia y amigos. Según evidencia revisada, el apoyo de los familiares es importante, especialmente en las etapas en que el paciente tiene dificultades para controlar las molestias físicas y emocionales en cáncer avanzado; una adaptación psicológica exitosa al cáncer está significativamente asociada al apoyo y comunicación con la familia y amigos ${ }^{(37,38)}$.

Otros resultados mostraron que existe un alto porcentaje de satisfacción de los participantes por el apoyo recibido de los profesionales de salud, especialmente del rol de la enfermera de la Unidad de Cuidados Paliativos. En este sentido, cobra relevancia la calidad de la atención desde la perspectiva humanizada e integral, por lo que se requiere fomentar aspectos humanos, espirituales y transpersonales en todas las funciones del rol de la gestión del cuidado ${ }^{(39,40)}$. Distintos autores afirman que las intervenciones de apoyo psicosocial asociada a una relación de comunicación cara a cara entre el paciente con cáncer y profesionales médico/ enfermera, se traduce en un efecto positivo en la calidad de vida para el paciente y familia ${ }^{(41,}$ ${ }^{42)}$. En este sentido, algunos autores recomiendan incorporar, en los aspectos terapéuticos, estrategias para el desarrollo de habilidades de afrontamiento, actitud positiva y comunicación efectiva entre paciente y profesionales de salud, con el fin de mejorar el afrontamiento psicológico del paciente a la enfermedad ${ }^{(43-45)}$. Con respecto al dominio Opción de participar en la elección del tratamiento, destaca que el 65,8\% de los participantes se sintió capaz y quería participar en ese aspecto, evidenciando un resultado que está en concordancia con un estudio realizado por los autores del instrumento original, Mystakidou et al. ${ }^{(24)}$. A este respecto, la evidencia muestra que los equipos médicos deben estar capacitados para preguntar en forma rutinaria a los pacientes con cáncer avanzado sobre sus deseos de atención y tratamiento, también argumentan que esta opción puede mejorar la adherencia al tratamiento $^{(35)}$. De esta manera, puede surgir el sentimiento subjetivo de estar adoptando decisiones compartidas al estar informado de todos los planes de tratamiento posibles y estrategias de autocuidado, disminuyendo así las preocupaciones relacionadas con el tratamiento ${ }^{(46)}$. Actualmente en Chile, aún no se ha considerado, en los pacientes con cáncer avanzado en cuidados paliativos, su participación en la selección o decisión del tratamiento.

La validez externa es relevante si se pretende realizar cuestionarios universales y si se busca su 
aplicación en otros ámbitos similares. Lamentablemente, la validez externa de la mayoría de los estudios es muy escasa, especialmente en lo que se refiere a llegar a conclusiones generalizadas ${ }^{(47)}$. Respecto al grado de generalización relativo a la validez externa, en este estudio pueden ser atribuidos inequívocamente a población que reúne las características y criterios similares en unidades de cuidados paliativos del sistema de salud, asimismo hay que considerar que el estudio es internamente válido.

Por otro lado, con los hallazgos obtenidos se pueden visibilizar recomendaciones de aplicación en un mayor número de población, basados en los resultados y la mejor evidencia disponible con el fin de completar la validez externa.

Respecto a las limitaciones del estudio, reconocemos que la principal radica en que la muestra seleccionada podría no ser lo suficientemente representativa de la población chilena con cáncer avanzado, ya que los participantes provienen de un solo hospital público de la ciudad de Santiago, por lo que no abarca a todo el espectro de pacientes beneficiarios de cuidados paliativos del país. En tanto, una segunda limitación puede ser la ausencia de un ítem relacionado a la caracterización de pertenencia a una etnia o pueblo originario determinado del paciente. Este ítem, considerado relevante en la atención médica en Chile, por las implicancias culturales, podría incidir en la percepción de la calidad de vida de las personas con cáncer avanzado, viéndose afectada así la validez externa del estudio ya que los resultados no podrían ser filtrados por este factor y se estaría generalizando a la totalidad de la población chilena con cáncer avanzado.

Los desafíos, en esta línea de investigación, están en la aplicación del instrumento en forma más representativa a nivel nacional, contemplando otras unidades de cuidados paliativos, tanto de Santiago como de otras regiones y localidades del país.

\section{CONCLUSIONES}

El presente estudio de adaptación transcultural y validación psicométrica del cuestionario PQLI, se llevó a cabo según metodología propuesta por expertos, con participación y validación de comité de expertos que permitieron dar consistencia al instrumento nuevo. Una vez aplicado en 155 participantes, de acuerdo a muestra descrita, este instrumento permitió obtener una visión global sobre la calidad de vida y la opción de participar en la elección del tratamiento, de los pacientes con cáncer avanzado de la Unidad de Cuidados Paliativos de un Hospital de Santiago de Chile. Si bien se deben considerar las limitaciones antes descritas, se determina que el instrumento resultante es coherente con el original de Mystakidou et al.

\section{REFERENCIAS}

1. The Lancet. GLOBOCAN 2018: counting the toll of cancer. Lancet [Internet]. 2018 Sep [citado 22 jun 2020]; 392(10152): 985. Disponible en: https://doi.org/10.1016/S0140-6736(18)32252-9

2. Ferlay J, Colombet M, Soerjomataram I, Dyba T, Randi G, Bettio M, et al. Cancer incidence and mortality patterns in Europe: Estimates for 40 countries and 25 major cancers in 2018. Eur J Cancer [Internet]. 2018 [citado 22 jun 2020]; 103: 356-87. Disponible en: https://onlinelibrary.wiley. com/doi/10.1002/ijc. 29210

3. Global Cancer Observatory: Cancer Today, International Agency for Research on Cancer Chile [Internet]. 2018 [citado 22 jun 2020]. Disponible en: https:/gco.iarc.fr/today/data/factsheets/popula tions/152-chile-fact-sheets.pdf

4. Jiménez de la Jara J, Bastías G, Ferreccio C, Moscoso C, Sagues S, Cid C, et al. A snapshot of cancer in Chile: analytical frameworks for developing a cancer policy. Biological res [Internet]. 2015 Ene [citado 22 jun 2020]; 48:10. Disponible en: https://doi.org/10.1186/0717-6287-48-10

5. Liegey Dougall A, Swanson J, Kyutoku Y, Belani CP, Baum A. Posttraumatic Symptoms, Quality of Life, and Survival among Lung Cancer Patients. J Appl Behav Res [Internet]. 2017 [citado 22 jun 2020]; 22(3): n/a-N.PAG. Disponible en: https:// doi.org/10.1111/jabr.12065

6. Looijmans M, Van Manen AS, Traa MJ, Kloover JS, Kessels BLJ, De Vries J. Psychosocial consequences of diagnosis and treatment of lung cancer and evaluation of the need for a lung cancer specific instrument using focus group methodology. Supportive care in cancer: official journal of the Multinational Association of Supportive Care in Cancer [Internet]. 2018 [citado 22 jun 2020]; 26(12): 4177-85. Disponible en: https://doi. org/10.1007/s00520-018-4291-1

7. Woten M, Heering H. Quality of Life Assessment: 
Administering the WHOQOL-OLD [Internet]. Pravikoff D, editor. CINAHL Nursing Guide EBSCO Publishing (Ipswich, Massachusetts), 2018 May 25 [citado 22 jun 2020]. Disponible en: https://research.ebscomedical.com/eds/detail?db= nup\&an=T904871\&isbn=\#citation-info

8. Razo GAM, Díaz CR, López GMP. Percepción del estado de salud y la calidad de vida en personas jóvenes, maduras y mayores. Rev CONAMED [Internet]. 2018 [citado 22 jun 2020]; 23(2): 5865. Disponible en: https://www.medigraphic.com/ cgi-bin/new/resumen.cgi?IDARTICULO $=80117$

9. Allemani C, Matsuda T, Di Carlo V, Harewood R, Matz M, Niksic M, et al. Global surveillance of trends in cancer survival 2000-14 (CONCORD-3): analysis of individual records for 37513025 patients diagnosed with one of 18 cancers from 322 population-based registries in 71 countries. Lancet [Internet]. 2018 Mar [citado 26 ago 2020]; 391(10125): 1023-75. Disponible en: https://doi. org/10.1016/S0140-6736(17)33326-3

10. Ministerio de Salud de Chile. MINSAL. "PLAN NACIONAL DE CÁNCER 2018-2028”. [Internet]. 2018 [citado 12 jul 2020]. p.185. Disponible en: https://www.minsal.cl/wp-content/ uploads/2019/01/2019.01.23_PLAN-NACIONAL -DE-CANCER_web.pdf

11. Moser EC, Meunier F. Cancer survivorship: A positive side-effect of more successful cancer treatment. EJC Suppl [Internet]. 2014 Jun [citado 12 jul 2020]; 12(1): 1-4. Disponible en: https:// doi.org/10.1016/j.ejcsup.2014.03.001

12. Shin WK, Song S, Jung SY, Lee E, Kim Z, Moon HG, et al. The association between physical activity and health-related quality of life among breast cancer survivors. Health Qual Life Outcomes [Internet]. 2017 Jun [citado 12 jul 2020]; 15(1): 132. Disponible en: https://doi.org/10.1186/ s12955-017-0706-9

13. Conrad R, Mücke M, Marinova M, Burghardt A, Stieber C, Cuhls H, et al. Measurement of Quality of Life in Palliative Care: Evidence for CriterionOriented Validity of a Single-Item Approach. J Palliat Med [Internet]. 2017 Jun [citado 12 jul 2020]; 20(6): 604-10. Disponible en: https://doi. org/10.1089/jpm.2016.0218

14. Knaul FM, Bhadelia A, Rodríguez NM, ArreolaOrnelas H, Zimmermann C. The Lancet Commission on Palliative Care and Pain Relieffindings, recommendations, and future directions. Lancet Glob Health [Internet]. 2018 Mar [citado 12 jul 2020]; 6(Supl. 1): S5-6. Disponible en: https://doi.org/10.1016/S2214-109X(18)30082-2

15. Ministerio de Salud de Chile. MINSAL. Guía Clínica Alivio del Dolor por Cáncer Avanzado y Cuida- dos Paliativos [Internet]. 2011 [citado 12 jul 2020]. Disponible en: http://www.bibliotecaminsal.cl/wp/ wp-content/uploads/2016/04/Alivio-del-Dolorpor-C\%C3\%A1 ncer-y-Cuidados-Paliativos.pdf. Spanish.

16. Haugland T, Wahl AK, Hofoss D, DeVon HA. Association between general self-efficacy, social support, cancer-related stress and physical healthrelated quality of life: a path model study in patients with neuroendocrine tumors. Health Qual Life Outcomes [Internet]. 2016 Ene [citado 12 jul 2020]; 14:11. Disponible en: https://doi. org/10.1186/s12955-016-0413-y

17. Bączyk G, Kwapisz U, Karpińska K. Quality of life patients after surgical treatment of laryngeal cancer. JMS [Internet]. 2017 Jun [citado 12 jul 2020]; 86(2): 127-33. Disponible en: https://jms.ump. edu.pl/index.php/JMS/article/view/134/195

18. Soares CFC, Maria BF, Da Silva EHE, Das Graças PG. ¿Are the nutritional status and tube feeding associated with quality of life in oncologic patients on palliative care? Demetra: Rio de Janeiro [Internet]. 2019 [citado 12 jul 2020]; 14: 1-14. Disponible en: https://doi.org/10.12957/ demetra.2019.38198

19. Lira MT, Caballero E. Adaptación transcultural de instrumentos de evaluación en salud: historia y reflexiones del porqué, cómo y cuándo. Rev med clin condes [Internet]. 2020 [citado 12 jul 2020]; 31(1):85-94. Disponible en: https://doi. org/10.1016/j.rmclc.2019.08.003

20. Hill J, Bird HA, Lawton CW, Wright V. The arthritis impact measurement scales: an anglicized version to assess the outcome of British patients with rheumatoid arthritis. $\mathrm{Br} \mathrm{J}$ Rheumatol [Internet]. 1990 Jun [citado 19 may 2021]; 29(3): 193-6. Disponible en: https://doi.org/10.1093/ rheumatology/29.3.193

21. Beaton DE, Bombardier C, Guillemin F, Ferraz MB. Guidelines for the process of crosscultural adaptation of self-report measures. Spine (Phila Pa 1976) [Internet]. 2000 Dic [citado 12 jul 2020]; 25(24): 3186-91. Disponible en: 10.1097/00007632-200012150-00014

22. Ramada-Rodilla JM, Serra-Pujadas C, DelclósClanchet GL. Cross-cultural adaptation and health questionnaires validation: revision and methodological recommendations. Salud Pública Mex [Internet]. 2013 Ene-Feb [citado 12 jul 2020]; 55(1): 57-66. Disponible en: https:// www.medigraphic.com/pdfs/salpubmex/sal-2013/ sal131g.pdf

23. Alexandre NMC, Guirardello EDB. Cultural adaptation of instruments utilized in occupational health. Rev Panam Salud Pública [Internet]. 2002 
[citado 12 jul 2020]; 11(2): 109-11. Disponible en: https://iris.paho.org/handle/10665.2/8675

24. Mystakidou K, Tsilika E, Kouloulias V, Parpa E, Katsouda E, Kouvaris J, et al. The Palliative Care Quality of Life Instrument (PQLI) in terminal cancer patients. Health Qual Life Outcomes [Internet]. 2004 Feb [citado 12 jul 2020]; 2:8. Disponible en: https://doi.org/10.1186/1477-75 25-2-8

25. Pedrozo-Pupo JC, Córdoba PA, Campo-Arias A. Estructura factorial y consistencia interna de la escala de somnolencia de Epworth. rev fac med [Internet]. 2020 Abr-Jun [citado 19 may 2021]; 68(2): 183-7. Disponible en: https://doi. org/10.15446/revfacmed.v68n2.73025.

26. Norman G, Streiner DL. Bioestadística. Barcelona: Mosby; 1996. pp. 129-148.

27. Ramírez-Arellano EE. Cuidados paliativos, ¿cuándo referir? Rev Sanid Milit Mex [Internet]. 2019 [citado 19 may 2021]; 73(2): 146-54. Disponible en: https://revistasanidadmilitar.org/index.php/rsm /article/view/23/24

28. Cronbach LJ. Coefficient alpha and the internal structure of tests. Psychometrika [Internet]. 1951 [citado 19 may 2021]; 16(3): 297-334. Disponible en: http://cda.psych.uiuc.edu/psychometrika_highly _cited_articles/cronbach_1951.pdf

29. Montoya SO. Aplicación del análisis factorial a la investigación de mercados. Caso de estudio. Scientia et Technica [Internet]. 2007 Ene [citado 12 jul 2020]; 1(35). Disponible en: https://revistas. utp.edu.co/index.php/revistaciencia/article/ view/5443

30. Tafernaberry G, Otero G, Agorio C, Dapueto JJ. Evaluation of the Charing Cross Venous Ulcer Questionnaire in patients with chronic venous ulcers in Uruguay. Rev Med Chil [Internet]. 2016 Ene [citado 12 jul 2020]; 144(1): 55-65. Disponible en: https://doi:10.4067/S0034-9887201600010 0008

31. Smith $\mathrm{CH}$, Graham CA, Herbet AR. Respite needs of families receiving palliative care. J Paediatr Child Health [Internet]. 2017 Feb [citado 12 jul 2020]; 53(2): 173-179. Disponible en: https://doi. org/10.1111/jpc. 13324

32. Aguiar-Palacios LH, Negrete-Cortés AJ, MartínezAlvarado JR, Magallanes RAG, García-Gomar ML. Propiedades psicométricas del inventario autoeficacia percibida para el control de peso en estudiantes universitarios del área de la salud. Nutr Hosp [Internet]. 2018 Ago [citado 12 jul 2020]; 35(4): 888-93. Disponible en: https://doi. org/10.20960/nh.1557

33. Costantini M, Rabitti E, Beccaro M, Fusco F, Peruselli C, La Ciura P, et al. Validity, reliability and responsiveness to change of the Italian palliative care outcome scale: a multicenter study of advanced cancer patients. BMC Palliat Care [Internet]. 2016 Feb [citado 23 sep 2020]; 15: 1-12. https://doi. org/10.1186/s12904-016-0095-6.

34. Sousa VD, Rojjanasrirat W. Translation, adaptation and validation of instruments or scales for use in cross-cultural health care research: a clear and userfriendly guideline. J Eval Clin Pract [Internet]. 2011 Abr [citado 23 sep 2020]; 17(2): 268-74. Disponible en: https://doi.org/10.1111/j.13652753.2010.01434.x

35. Garcimartin P, Comin-Colet J, Delgado-Hito P, Badosa-Marcé N, Linas-Alonso A. Transcultural adaptation and validation of the patient empowerment in long-term conditions questionnaire. BMC Health Serv Res [Internet]. 2017 May [citado 10 ago 2020]; 17(1): 324. Disponible en: https://doi.org/10.1186/s12913-017-2271-7

36. Criado-Álvarez JJ, González González J, Martín García S, Romo Barrientos C. Quality of life in patients with oral anticoagulation therapy. Med Clin (Barc) [Internet]. 2015 Ene [citado 10 ago 2020]; 144(1): 46-7. Disponible en: https://doi. org/10.1016/j.medcli.2014.02.011

37. Kim YH, Choi KS, Han K, Kim HW. A psychological intervention programme for patients with breast cancer under chemotherapy and at a high risk of depression: A randomised clinical trial. J Clin Nurs [Internet]. 2018 Feb [citado 10 ago 2020]; 27(3-4): 572-81. Disponible en: https://onlinelibrary.wiley.com/doi/full/10.1111/ jocn. 13910

38. Salonen P, Tarkka MT, Kellokumpu-Lehtinen PL, Koivisto AM, Åstedt-Kurki P, Kaunonen M. Individual face-to-face support and quality of life in patients with breast cancer. Int J Nurs Pract [Internet]. 2011 Ago [citado 10 ago 2020]; 17(4): 396-410. Disponible en: https://doi.org/10.1111/ j.1440-172X.2011.01948.x

39. Meleis AI. Theoretical nursing Development \& progress. $5^{\mathrm{a}}$ ed. Filadelfia: Lippincott Williams \& Wilkins; 2012. 672 p.

40. Watson J. Watson's theory of human caring and subjective living experiences: carative factors/caritas processes as a disciplinary guide to the professional nursing practice. Texto contexto - enferm [Internet]. 2007 [citado 20 ago 2020]; 16(1): 129-135. Disponible en: https://doi.org/10.1590/ S0104-07072007000100016

41. Son H, Son Y-J, Kim H, Lee Y. Effect of psychosocial interventions on the quality of life of patients with colorectal cancer: a systematic review and meta-analysis. Health Qual Life Outcomes [Internet]. 2018 Jun [citado 10 ago 2020]; 16(1): 
119. Disponible en: https://doi.org/10.1186/s12 955-018-0943-6

42. Yoo SH, Kim M, Yun YH, Keam B, Kim YA, Kim $\mathrm{YJ}$, et al. Attitudes toward early palliative care in cancer patients and caregivers: a Korean nationwide survey. Cancer Med [Internet]. 2018 May [citado 10 ago 2020]; 7(5): 1784-93. Disponible en: https://onlinelibrary.wiley.com/doi/full/10.1002/ cam4.1441

43. D'Ambruoso SF, Coscarelli A, Hurvitz S, Wenger $\mathrm{N}$, Coniglio D, Donaldson D, et al. Use of a Shared Mental Model by a Team Composed of Oncology, Palliative Care, and Supportive Care Clinicians to Facilitate Shared Decision Making in a Patient With Advanced Cancer. J Oncol Pract [Internet]. $2016 \mathrm{Nov}$ [citado 10 ago 2020]; 12(11): 1039-45. Disponible en: https:// doi:10.1200/ JOP.2016.013722.

44. Zimmermann C, Swami N, Krzyzanowska M, Hannon B, Leighl N, Oza A, et al. Early palliative care for patients with advanced cancer: a cluster- randomised controlled trial. Lancet [Internet]. 2014 May [citado 10 ago 2020]; 383(9930): 1721-30. Disponible en: https://doi.org/10.1016/ S0140-6736(13)62416-2

45. González TM, Bilbao A, Baré M, Briones E, Sarasqueta C, Quintana JM, et al. Association of social support, functional status, and psychological variables with changes in health-related quality of life outcomes in patients with colorectal cancer. Psychooncology [Internet]. 2016 Ago [citado 10 ago 2020]; 25(8): 891-7. Disponible en: https:// www.chicom.be/sites/default/files/tejada_et_al2016-psycho-oncology.pdf

46. Jitender S, Mahajan R, Rathore V, Choudhary R. Quality of life of cancer patients. J Exp Ther Oncol. 2018 may [citado 10 ago 2020]; 12(3): 217-221.

47. Ioli P. Validez externa de los ensayos clínicos en Neurología. Neurol Arg [Internet]. 2011 Ene [citado 19 may 2021]; 3(1): 54-60. Disponible en: https://doi.org/10.1016/S1853-0028(11)70007-6 